\title{
Contextual control of conditioning is not affected by extinction in a behavioral task with humans
}

\author{
James Byron Nelson • Jeffrey A. Lamoureux
}

Published online: 19 March 2015

(C) Psychonomic Society, Inc. 2015

\begin{abstract}
The Attentional Theory of Context Processing (ATCP) states that extinction will arouse attention to contexts resulting in learning becoming contextually controlled. Participants learned to suppress responding to colored sensors in a video-game task where contexts were provided by different gameplay backgrounds. Four experiments assessed the contextual control of simple excitatory learning acquired to a test stimulus (T) after (Exp. 1) or during (Exp. 2-4) extinction of another stimulus (X). Experiment 1 produced no evidence of contextual control of $\mathrm{T}$, though renewal to $\mathrm{X}$ was present both at the time $T$ was trained and tested. In Experiment 2 no contextual control of $\mathrm{T}$ was evident when $\mathrm{X}$ underwent extensive conditioning and extinction. In Experiment 3 no contextual control of T was evident after extensive conditioning and extinction of $X$, and renewal to $X$ was present. In Experiment 4 contextual control was evident to $\mathrm{T}$, but it neither depended upon nor was enhanced by extinction of X. The results presented here appear to limit the generality of ATCP.
\end{abstract}

Keywords Associative learning $\cdot$ Attention $\cdot$ Context . Extinction $\cdot$ Human learning $\cdot$ Renewal

Research investigating predictions of the Attentional Theory of Context Processing (ATCP, Rosas and Callejas-Aguilera

J. B. Nelson

University of the Basque Country (UPV/EHU), San Sebastián, Spain

J. A. Lamoureux

Boston College, Chestnut Hill, MA, USA

J. B. Nelson $(\bowtie)$

Departamento Procesos Psicológicos Básicos y su Desarrollo, Universidad de País Vasco (UPV/EHU), Avenida de Tolosa, 70, San Sebastián, España 20018

e-mail: JamesByron.Nelson@ehu.es
2006; Rosas et al. 2006a, b) has routinely demonstrated that extinction conducted with one stimulus can lead to contextual control of learning about other stimuli. To illustrate, participants might initially learn that a food cue " $\mathrm{X}$ " predicts that fictitious patrons will get sick in the context of a certain restaurant. Then, they learn that $\mathrm{X}$ no longer produces illness; patrons consume $\mathrm{X}$ without consequence. During this second phase where extinction occurs, the participants also learn that a new cue " $T$ " predicts illness. Here, we have two cues; one cue $\mathrm{X}$ has been conditioned and extinguished, the other cue $\mathrm{T}$ has simply been conditioned while the $\mathrm{X}$ was undergoing extinction. Each cue is then tested in a different context to where it was learned. The general result is "renewal" (e.g., Bouton and Bolles 1979; Nelson et al. 2011a) with X, and a loss of response to T. Participants rate $\mathrm{X}$ as more likely to predict illness in the new context than in the training context where extinction took place, and rate $\mathrm{T}$ as less likely to produce the illness in the new context than in the training context. The result with $\mathrm{T}$ is the one of particular interest. A control condition that receives only conditioning of $\mathrm{T}$, without extinction of X, shows no effect of a context change. Simple conditioning of $\mathrm{T}$ is not much affected by a context change. Contextual control of what was being learned about both $\mathrm{X}$ and $\mathrm{T}$ emerged during extinction of X.

The result is predicted by ATCP as the theory assumes that contextual control will emerge whenever attention is directed to contexts, and a major contribution of the theory is explicitly specifying the conditions under which such attention will be aroused. The condition of most relevance to the research presented here is the presence of "ambiguity." In this case the theory borrows from Bouton (e.g., 1993, 1997) in assuming that when a stimulus is paired with different outcomes, multiple associations are formed (e.g., $X \rightarrow$ Illness, $X \rightarrow$ No Illness) leaving the stimulus as functionally ambiguous. Like Bouton (1997), ATCP assumes that attention to the context is aroused in the presence of ambiguity. The additional assumption made 
by ATCP is that it is this attention that results in contextual control. Moreover, according to ATCP, attention to contexts results in contextual control not only of the learning that produced the ambiguity, but any learning that occurs in that context while attention is aroused. Thus, renewal occurs to $\mathrm{X}$ and a loss of predictive ratings occur to $\mathrm{T}$ because the surprise and ambiguity produced by the absence of illness in the second phase aroused attention to the context, resulting in the contextual control of both the new interfering $X \rightarrow$ No Illness learning and the simpler $\mathrm{T} \rightarrow$ Illness learning. According to ATCP it is not that contextual control necessarily favors secondlearning (Bouton 1993; Nelson 2002, 2009) or interfering information (Nelson and Callejas-Aguillera 2007), but it simply favors any learning that occurs in a context that is receiving attention.

Nelson et al. (2011b) reiterate the well appreciated fact that the renewal effect and its explanatory constructs are of extensive relevance to psychology as they concern any issue where multiple things might be learned about a single event (see Bouton 1993, 2004, for review). The effect and its underlying constructs have application to diverse phenomena such as emotional intelligence (Nelson and Bouton 2002), perceptual learning (e.g., Nelson and Sanjuan 2008, 2009), and emotional regulation (Nelson 2013). Thus the ATCP, which has been successfully applied to issues in social psychology (e.g., Gawronski and Cesario 2013; Gawronski et al. 2010; Ye and Gawronski 2014), is one of potentially great breadth.

With humans, the contextual control of simple conditioning that is learned in a context where extinction has taken place has been replicated in different laboratories (e.g., Nelson and Callejas-Aguillera 2007). These demonstrations have all used predictive-learning scenarios that were based on, and almost identical to, those of Rosas and Callejas-Aguilera 2006. With animals, the effect has been demonstrated in tasks that have involved, in one form or another, the use of liquids as conditioned stimuli (CSs) or reinforcers in thirsty rats (BernalGamboa et al. 2013, 2014; Rosas and Callejas-Aguilera 2007). However, using food reinforcers in hungry rats, Nelson et al. (2011a) found no evidence of a loss in responding to a simple excitatory CS with a context switch when that responding was acquired while another CS was being extinguished. Thus, the generality of the account offered by ACTP with animals is somewhat tentative. To date, there has been no demonstration of the effect with humans outside of predictive-learning type tasks.

Given the potential scope of the theory, particularly with respect to humans where the theory has been extended to automatic evaluative processes in social learning (also using procedures that largely mirror those found in predictivelearning tasks; Gawronski et al. 2010), it is important to determine whether the basic effect, contextual control generally arising as a function of extinction, can be demonstrated in other methods. For the present research we chose the behavioral suppression task developed by Nelson and Sanjuan (2006). The task is a video game where participants learn to suppress their baseline rate of mouse-clicking in preparation for unavoidable attacks from an enemy spacecraft in the presences of sensors. The task has successfully demonstrated contextual control of extinction (i.e., renewal) in the absence of evidence for direct context-outcome associations (Nelson et al. 2011b) as well as context-specificity of both latent inhibition (Nelson and Sanjuan 2006) and perceptual learning (Nelson and Sanjuan 2008). Importantly, the task has been used to provide evidence that attention to contexts is aroused by extinction (Nelson et al. 2013).

A criticism of the work regarding ATCP first acknowledged by Rosas and Callejas-Aguilera (2006) and raised again by Nelson et al. (2013) is that there has been no independent demonstration that extinction encourages attention to contexts aside from the result for which aroused attention is invoked to explain. To address that concern, Nelson et al. used the suppression task used in the present experiments and gave participants conditioning trials where a purple sensor predicted an attack. Half of the participants then received extinction with that sensor. Following that phase, both groups received a biconditional discrimination in the form RG+/BG-/RY-/BY+. For a third of each group, the contexts where the gameplay was taking place were correlated with the trial sequences such that participants might receive $\mathrm{RG}+\mathrm{BG}-$ in Context $\mathrm{A}$ and $\mathrm{RY}-\mathrm{BY}+$ in Context B. If the context commands attention then participants only need to learn to respond to sensor $\mathrm{R}$ in Context A and sensor B in Context B. Two other groups received all trial types in each context, and the context was irrelevant to the solution. The experiment was, thus, a factorial where the presence or absence of prior extinction was combined with whether or not the identities of two contexts were correlated with the solution to a difficult problem. If extinction arouses attention to the contexts then participants should better notice the correlation of the context with the solution and solve the discrimination more rapidly than participants that did not receive extinction, or those for which the context was irrelevant to the solution. The resulting data supported the idea that attention had been aroused to the contexts. Extinction facilitated learning the bi-conditional discrimination when the contexts were correlated with the solution. The method produces renewal (e.g., Nelson et al. 2011a) and evidence that extinction arouses attention to the contexts. The method is ideal for determining whether or not contextual control of simple conditioning will emerge when that conditioning is conducted in the face of ambiguity produced by the extinction of another CS with humans outside of the tasks used thus far in the literature.

Our goal was not to refute ATCP, but to demonstrate its predictions regarding contextual control of simple conditioning after extinction in a different method. We began with the full expectation of observing the effect, and with the result in 
hand we could then explore the boundary conditions under which it occurs to further elaborate the theory. Our initial work failed to produce the effect, leading to subsequent attempts. In those attempts we manipulated variables that should enhance our chances of observing the effect yet each experiment failed to do so.

\section{Experiment 1}

In the study by Nelson et al. (2013), evidence for attention to the context was obtained after conditioning and extinction of one sensor. The present experiment used the same parameters as were used in the first two attention-arousing phases of that study. The design is shown in Table 1. Participants in Groups With Extinction and No Extinction received conditioning with $\mathrm{X}$, followed by extinction of $\mathrm{X}$ in Group With Extinction, or not in the case of Group No Extinction. Then, each of those groups received conditioning with $\mathrm{T}$ and testing in Context $\mathrm{B}$, a different context from where training took place. The prediction here is clear. Extinction with $\mathrm{X}$ should arouse attention to the context such that conditioning of $\mathrm{T}$ will be context specific, resulting in less suppression to $\mathrm{T}$ in Group With Extinction than Group No Extinction in the test context.

There is considerable evidence that context changes with the method are easily discernible (Nelson and Sanjuan 2006, 2008; Nelson et al. 2011b; Nelson et al. 2012, 2013), nevertheless we included four additional groups to ensure that is the case with the present samples and parameters. All four of these groups received conditioning and extinction of $\mathrm{X}$ in the same way as did Group With Extinction. Two of the four groups formed Group Renewal Before and were tested with $\mathrm{X}$ in Context $\mathrm{A}$ where it had just been extinguished or in Context $\mathrm{B}$ where it had never before been presented. An "AAB" renewal effect was expected here, demonstrating that extinction learning had come under the control of the context, and that the context change was effective at the time participants in the Extinction/No Extinction Groups were

Table 1 Design of Experiment 1

\begin{tabular}{|c|c|c|c|c|c|}
\hline Group & $\begin{array}{l}\text { Context B } \\
\text { (exposure) }\end{array}$ & Phase 1 & Phase 2 & Phase 3 & Test \\
\hline No Extinction & \multirow{6}{*}{ B: -- } & \multirow{6}{*}{ A: $8 \mathrm{X}+$} & A: -- & A: $5 \mathrm{~T}+$ & B: $T$ \\
\hline With Extinction & & & \multirow{5}{*}{ A: $8 X-$} & A: $5 \mathrm{~T}+$ & B: T \\
\hline Renewal After-A & & & & A: $5 \mathrm{~T}+$ & A: $X$ \\
\hline Renewal After-B & & & & A: $5 \mathrm{~T}+$ & B: $X$ \\
\hline Renewal Before-A & & & & (direct to test) & A: $X$ \\
\hline Renewal Before-B & & & & (direct to test) & B: X \\
\hline \multicolumn{6}{|c|}{$\begin{array}{l}\text { Note A: and B: are different galaxy contexts. } \mathrm{X} \text { is a purple light, } \mathrm{T} \text { is a } \\
\text { yellow light. + and - refer to the presence and absence of an attack by an } \\
\text { enemy spaceship, respectively. Numbers refer to the number of trials. } \\
\text { indicates exposure to the context }\end{array}$} \\
\hline
\end{tabular}

undergoing conditioning with $\mathrm{T}$. The final two groups received conditioning with $\mathrm{T}$ as did the Extinction/No Extinction Groups, but were tested with $\mathrm{X}$ in contexts $\mathrm{A}$ or B. These groups should also show an AAB Renewal effect, confirming that the context change was effective at the time that $\mathrm{T}$ was being tested in the Extinction/No Extinction Groups.

Method

\section{Subjects}

Subjects were 144 students recruited from college campuses. Conditions were randomly assigned to participants without replacement until each condition had been assigned once to place 24 participants in each condition. An assignment error resulted in one participant intended for Group Renewal After A $(n=23)$ being run with the Renewal After B $(n=25)$ protocol.

\section{Apparatus}

The same apparatus as was used in Nelson and Sanjuan (2006, 2008, 2009; Nelson et al. 2011a; Nelson et al. 2012, 2013) was used here. The apparatus has been thoroughly described in Nelson and Sanjuan (2006) and the description here is adapted from Nelson and Sanjuan (2008) with permission. Participants received written instructions that they were playing a game in which they were to earn points by shooting torpedoes at an on-screen spaceship by clicking the mouse. They were further instructed that sometimes they would be attacked and that the attack would damage their spaceships by draining their power, leaving the participants unable to continue the game until power was recharged. They were instructed that they could not avoid the attack, but that they could prepare for it by conserving their power (suppressing their own rate of torpedo firing) when they believed they were about to be attacked. They were told that sensors would appear that might help them in the game, and they were not told what the sensors would indicate. The instructions were the same as those reported in detail in Nelson and Sanjuan (2006).

The video game was viewed on 35.56-cm (14-in) Dell laptop computer monitors $(30.5 \mathrm{~cm} \times 19 \mathrm{~cm} \mathrm{~W} / \mathrm{H})$. The resolution was set to $800 \times 600$ pixels and 24-bit color depth. On the monitor, an image was presented so that it was as if the participant was sitting inside of a spaceship looking out of a viewscreen. The viewscreen was a rectangular window that was 618 pixels wide and 368 pixels tall and was centered from left to right, approximately 91 pixels below the top of the screen on a gray metallic background. A box appeared approximately 34 pixels below the top of the screen in which the word "Points" appeared in yellow. At the bottom of the screen, five black ovals appeared that were each 84 pixels in 
diameter. The third was centered from left to right and was located approximately 21 pixels above the bottom of the screen. The other four ovals were spaced at intervals of approximately 52 pixels to the left and right of the center oval. One of two colored backgrounds (Hubble Space Telescope photos of the Eagle 1 [Pillars of Creation area] or Crab Nebulae) could be seen through the viewscreen on which a threedimensional representation of a spaceship was flying in a randomly determined path. These colored backgrounds provided contexts and were always counterbalanced. Sensor stimuli were the illumination of the ovals. $\mathrm{X}$ was the 5-s illumination of the second oval with the color purple (red, green, and blue components as $\mathrm{R}=255, \mathrm{G}=0$, and $\mathrm{B}=255$, respectively) and $\mathrm{T}$ was the illumination of the third oval with the color yellow $(\mathrm{R}=255, \mathrm{G}=255, \mathrm{~B}=0)$. The hypothetical "unconditioned stimulus" was presented in the form of an inescapable attack from the enemy spacecraft. Immediately upon the offset of a sensor stimulus, a round green torpedo emerged from the onscreen spacecraft and exploded in the center of the viewscreen. The message "Power at _ percent. Controls frozen for ___ seconds." appeared in the center of the viewscreen and remained until "Power" incremented to 100 and "Controls frozen for_" decremented to 0 (changes occurring roughly every second). During this time, the computer mouse was inoperable, and actions of the participant were not reflected on the screen. The numbers in the blanks were determined by a suppression ratio in which the number of clicks during the $5 \mathrm{~s}$ prior to the attack (i.e., during the presentation of the sensor) was divided by that number plus the average rate of mouse clicks in the $5 \mathrm{~s}$ before stimulus presentations across the game. The resulting ratio was then multiplied by 120 . For example, if a participant clicked, on average, ten times prior to the sensor CS and did not suppress his or her rate of clicking (clicking ten times during the sensor CS), then the ratio would calculate to .5 , and their controls would be frozen for $60 \mathrm{~s}$ by an attack.

Context changes were initiated by presenting a standard Microsoft Windows message box with the text "Please attend to this important message" displayed on the title bar of the message box. The text in the message box read, "You, your sensors, and the enemy are being transported to

galaxy for further testing. Press 'OK' to proceed." When the participant pressed the "OK" button, another message box was displayed. The text "Press 'OK' now for immediate transport" was displayed in the title bar, and the text in the message box read, "Remember, you, your sensors, and the enemy are being transported to galaxy." Fictitious names of the galaxies (e.g.,Blue Galaxy, Crab Galaxy) were presented where indicated by the blanks. When the participant pressed the "OK" button, the screen flickered, and the current background (e.g., Crab Nebula) was replaced with the alternative (e.g., Eagle 1 Nebula).

\section{Procedure}

The experiment began with $310 \mathrm{~s}$ of exposure to Context B where participants played the game with no stimuli being presented. Then, they were switched to Context A where they began Phase 1 and received eight conditioning trials with $\mathrm{X}$ with a variable intertrial interval (ITI) averaging $9.5 \mathrm{~s}$. These parameters were exactly the same as in the analogous phase of Nelson et al. (2013). Following conditioning with X, all groups entered Phase 2. Except for Group No Extinction, all groups received eight extinction trials where $\mathrm{X}$ appeared without an attack. Group No Extinction received the same trials but the $\mathrm{X}$ stimulus was presented as black, producing no visual changes in the game. Extinction of $X$ followed the same parameters as were used in the analogous phase in Nelson et al. (2013). The ITI was again variable with a mean of 9.5 s. Following phase 2, Groups Renewal Before A and Renewal Before $\mathrm{B}$ received a test with $\mathrm{X}$ either in Context A or Context B, respectively. There were four test trials with an average ITI of $10.5 \mathrm{~s}$. The experiment ended for these two groups. The remaining groups entered Phase 3 and received five conditioning trials with $\mathrm{T}$ in Context $\mathrm{A}$ with an average ITI of $10 \mathrm{~s}$. Following the conditioning of $\mathrm{T}$ all remaining groups were tested. The Renewal After A and Renewal After $\mathrm{B}$ groups were tested with $\mathrm{X}$ in Contexts $\mathrm{A}$ and $\mathrm{B}$, respectively. The Extinction and No Extinction Groups were tested with $\mathrm{T}$ in Context B. The ITIs on these trials were the same as those used on test in the Renewal Before Groups.

\section{Data analysis}

The data analysis strategy described here shall apply to all experiments reported in this manuscript. Responses were recorded during the 5-s CS and during the 5-s immediately preceding the CS (pre-CS). These data were converted into standard suppression ratios (CS / (pre-CS + CS)). Participants were initially screened to remove those who failed to learn the basic task during the first phase. Participants for whom suppression ratios on two out of the last three trials of the first phase of conditioning were not lower than the first trial were to be excluded (see also Nelson et al. 2011b). In previous reports using this method (e.g., Nelson et al. 2011a, 2012) we excluded participants who had low pre-CS responding (average $<5$ ) as that procedure was successful in eliminating the occasional pre-CS response rate of zero for which calculation of a suppression ratio would be uninterpretable. However, in these experiments that criterion did not meet that goal successfully. Therefore, participants that had a zero pre-CS rate on any of the variables in a particular analysis were eliminated from that analysis. Chi-square tests of independence were conducted at each step to assess whether or not the exclusions produced any selection bias, and are reported here only in 
the one case where the test was significant. Where not reported, there was no relationship between being excluded and group membership.

Suppression ratio data were analyzed with mixed (within-between) analysis of variance (ANOVA) using type-3 (unweighted) sums of squares. Simple effect tests were also conducted with ANOVA using error terms and degrees of freedom derived from the overall ANOVA using standard procedures as described by Howell (1987). Effect sizes are reported as partial-eta squared for ANOVAs with more than one independent variable, and as Cohen's $d$ for any ANOVA involving only two means, using the pooled variance around the two means being compared.

For null-results that go against the hypothesis, the odds of the null given the data, relative to the alternative, were computed using the Bayesian methods described by Wagenmakers (2007; see also Masson 2011). This method, based on the Baeysian Information Criterion (BIC, see Glover and Dixon 2004, for other example usages) was chosen for its computational simplicity and the reasonable objective practice of assigning an equal prior probability to the models specified by the null (i.e., $x=\mu$ ) and the alternative (e.g., $x=\mu+$ Context + Extinction + Extinction $\times$ Context). The BIC is a statistic that adjusts a standard likelihood ratio (i.e., comparing the likelihood of one model of the data to another) according to the complexity of the model. In the present work it was calculated as $n \ln \left(\frac{S S \text { Error }}{S S \text { Effect }+S S \text { Error }}\right)+\operatorname{Ln}(n) D F$ effect and represents the improvement in fit of the data by the alternative model over the null. The resulting BIC (or $\triangle B I C$ as it represents a difference between the null and alternative models) was then transformed into an estimate of the Bayes factor by $e^{.5 B I C}$, representing the posterior odds of the null being the true state of affairs over the alternative.

Above, $n$ represents the number of subjects for both between- and within-subject factors. The issue of $n$ in the within-subject case is a subject of debate (see Masson 2011). It can represent the number of subjects $(\mathrm{N})$ or be linked to the number of observations $(\mathrm{N} *$ (within subject factors -1 )). Lindley has shown that when a result is significant at some alpha level, a sample size $(\mathrm{N})$ can be found such that the posterior probability of the null is nevertheless 100 minus alpha (i.e., Lindley's paradox, Lindley 1957). With increases in $\mathrm{N}$ the evidence favoring the null hypothesis increases. For that reason $n$ in the calculation of the BIC was here chosen consistently as the number of subjects to provide more conservative estimates of the support for the null hypothesis. The strength of support offered by those odds was interpreted using guidelines derived from Raftery (1995; as discussed in Wagenmakers 2007) as weak (1-3), positive (3-19), strong (19-99), and very strong $(>99)$.

\section{Results}

The data from the first three phases are shown in Fig. 1, which collapses across the six grouping variables as there were no group differences. All groups acquired suppression to X during Phase 1 and those that received extinction showed decreases in suppression during Phase 2 extinction. Suppression to $T$ was acquired in the Groups that received conditioning of T during Phase 3. On test, shown in Fig. 2, there was evidence of AAB renewal when the extinguished $\mathrm{X}$ was tested, but there was no effect of prior extinction of $\mathrm{X}$ on responding to $\mathrm{T}$ in Context B.

\section{Phases 1- 3}

Group $\times$ Trials ANOVAs were conducted on each phase. In Phase 1 all six groups were included. Phase 2 included the five groups that received extinction. Phase 3 included all groups except the Renewal Before conditions as they were retired prior to this phase. Data screening eliminated six participants in Phase 1, 11 in Phase 2, and two in Phase 3. There were effects of Trials in each phase, with suppression to $\mathrm{X}$ increasing in Phase $1, F(7,833)=147.91, p<.0001, \eta_{p}^{2}=.55$, decreasing in Phase 2, $F(7,644)=43.11, p<.0001, \eta_{p}^{2}=$ .32 , and increasing to $\mathrm{T}$ in Phase $3, F(4,332)=18.77, p<$ $.0001, \eta_{p}^{2}=.18$. In no case were there effects of, or involving, the grouping variable, $p \mathrm{~s} \geq .13$.

\section{Testing}

The data from the tests are shown in Fig. 2. The left-most panel shows the renewal tests where the extinguished $\mathrm{X}$ was tested in Context A where conditioning and extinction had occurred, or in Context B where it had never before been

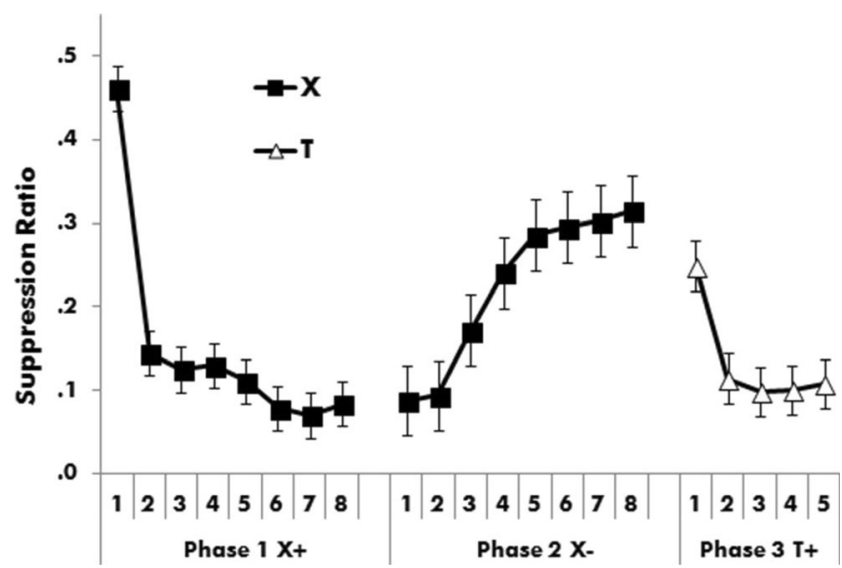

Fig. 1 Suppression to $X$ during Phase 1 conditioning, Phase 2 extinction, and suppression to $\mathrm{T}$ during Phase 3 conditioning during Experiment 1. + indicates trials where the conditioned stimulus (CS) was paired with an attack. - indicates trials without an attack. Bars represent the standard error of the mean 


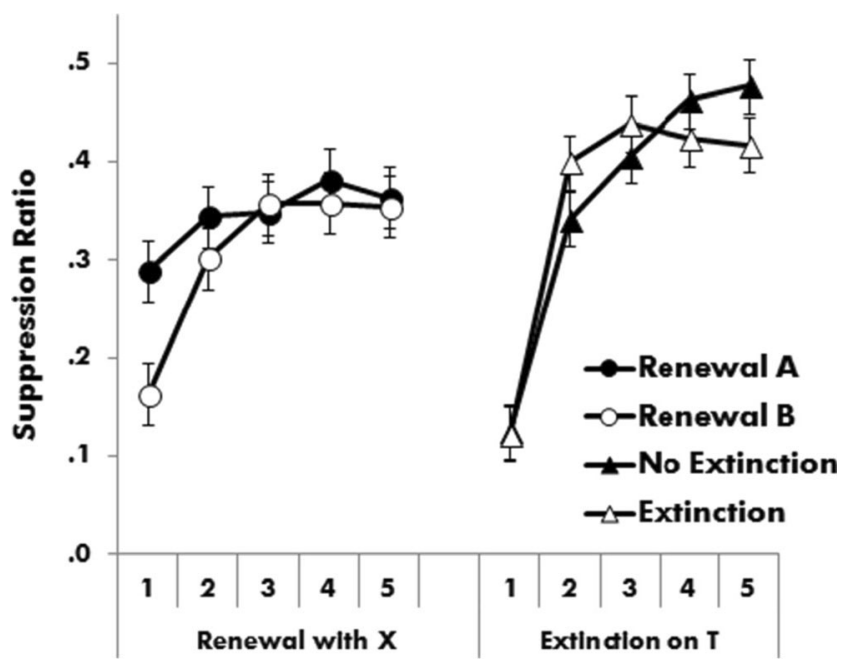

Fig. 2 Test data from Experiment 1. The left panel shows suppression to $\mathrm{X}$ test in Context A (solid circles) where extinction of X had occurred or Context B (open circles) where $\mathrm{X}$ had never before been presented. The right panel shows responding to $\mathrm{T}$ in Context $\mathrm{B}$ where it had never before been presented. Solid triangles represent groups for which $\mathrm{T}$ was conditioned without prior extinction of $\mathrm{X}$ occurring. Open triangles represent groups for which $\mathrm{T}$ was conditioned after $\mathrm{X}$ had been extinguished. Bars represent the standard error of the mean. See text for details

presented. The right panel shows the effect of prior Extinction of $\mathrm{X}$ on suppression to $\mathrm{T}$ in Context $\mathrm{B}$. The analyses support that there was a renewal effect, and there was no effect of prior Extinction of $\mathrm{X}$ on responding to $\mathrm{T}$ in Context $\mathrm{B}$.

Renewal testing Responding to $\mathrm{X}$ was analyzed with a Test Time (before or after Phase 3 ) by Context (same/different) $\times$ Trials ANOVA. The data are shown at left in Fig. 2, which collapses across the Test Time variable as it did not affect the Context effect. There were effects of Trials and Trials by Test Time, $F \mathrm{~s}(4,316) \geq 2.54, p \mathrm{~s} \leq .04, \eta_{p}^{2}=.22$ and .05 , respectively. Regarding the interaction, suppression to the extinguished $\mathrm{X}$ during the first few trials was somewhat greater after Phase 3 than before it, allowing for greater extinction of responding (i.e., the effect of trials) in the later test.

Of most interest, there was a Context $\times$ Trials interaction, $F(4,316)=4.53, p=.001, \eta_{p}^{2}=.05$ that did not interact with Test Time, $F<1$. Collapsed over trials, the Context $\times$ Test Time interaction approached reliability, $F(1,79)=.08$. Because suppression on the later test was initially somewhat greater, the effect of context tended to persist longer (i.e., it extinguished into a ceiling more slowly) producing an insignificant trend for a larger context effect at the later test. Simple effect tests of Context on each trial showed an effect on Trial $1, F(1,136)=8.12, p=.005, d=1.28$. There were no differences on any other trial, $F_{\mathrm{S}}<1$.

Effect of extinction on $T$ in $B$ Responding to T was analyzed with an Extinction (preceded by Extinction of $\mathrm{X}$, or not) $\times$
Trials ANOVA. These data are shown in the right panel of Fig. 2. There was an effect of Trials, $F(4,168)=54.06, p<$ $.0001, \eta^{2}{ }_{p}=.56$. There was no effect of Extinction, $F<1$, and no interaction $F(4,168)=1.74, p=.14$. The odds positively supported the null over the alternative with regards to the effect of Extinction (odds $=6.62$ ). Given the characteristics of the data we would expect that out of every 7-8 experiments an effect of Extinction might be observed once with null results obtained otherwise. The odds strongly supported the lack of an Extinction $\times$ Trials interaction (odds $=792.49$ ).

Due to our a-priori interest in the effect of Extinction, we nevertheless tested for an Extinction effect at each trial and found none, $F \mathrm{~s}(1,98) \leq 1.23, p \mathrm{~s} \geq .27$. The odds positively supported the null in every comparison (odds $\mathrm{sin}_{\text {min }}$ $\max =3.51-6.62$ ).

\section{Discussion}

To test the effect of prior extinction on contextual control of responding to a simple CS, $\mathrm{X}$ was extinguished or not prior to conditioning of $\mathrm{T}$. We used parameters that have produced independent evidence consistent with attention-like processes being aroused to contextual stimuli (Nelson et al. 2013) following extinction. That manipulation, according to ATCP, should make responding to $\mathrm{T}$ more context specific than it ordinarily might be. When tested in Context B, there was no evidence whatsoever of an effect of prior extinction. Moreover, the data provided positive support for the null hypothesis.

Other groups received simple conditioning and extinction of X. In some groups $\mathrm{X}$ was then tested in the context where conditioning and extinction had occurred, while other groups were tested in a different context, Context B. Testing occurred either at the time $\mathrm{T}+$ training was occurring in the With- and No-Extinction Groups, or afterwards. There was an effect of Context that did not depend upon test time where suppression to $\mathrm{X}$ was renewed. Thus, both at the moment that $\mathrm{T}+$ training was being conducted and when $\mathrm{T}$ was being tested there was evidence that the contexts could be discriminated. To the extent that such a result depends on attention to the context, as specified by ATCP, there was evidence that subjects were attending to the context. Despite that evidence, there was no observable effect of the extinction manipulation when $\mathrm{T}$ was tested in a different context.

In this experiment, extinction was incomplete. Inspection of Fig. 1 shows that the level of suppression on trial 8 of extinction was several standard errors below that of the first trial of conditioning. One interpretation of that level of responding is that it should reflect uncertainty about the meaning of $X$, and thus yield attention to the context as was supposedly produced by the same manipulation in Nelson et al. (2013). However, in another sense, the CS might not have been fully ambiguous. The level of responding might 
represent variation in the strength to which the CS predicts the attack, rather than competition between the extent to which the $\mathrm{CS}$ predicts an attack versus its absence. The acquisition of the second meaning might not have been fully realized (nevertheless it was sufficient to induce contextual control, as evidenced by the response recovery to X). Although there was still evidence of attention to the contexts as defined by ATCP in the Renewal Groups, the contextualization process may have ended with the extinction of $\mathrm{X}$ and been unable to affect $\mathrm{T}$.

\section{Experiment 2}

Experiment 2 was undertaken to ensure that $\mathrm{X}$ could become fully ambiguous, and that $\mathrm{T}$ was trained both during and after the production of that ambiguity. The design is shown in Table 2. In Experiment 1 we followed conditioning and extinction of $\mathrm{X}$ with conditioning of $\mathrm{T}$ because we had evidence that attention to contexts was present after extinction (Nelson et al. 2013). In Experiment 2, rather than completing the extinction phase with $\mathrm{X}$ and then conditioning with $\mathrm{T}$, we used the procedure used in the majority of the human demonstrations where $\mathrm{T}$ is conditioned concurrently with the extinction of $\mathrm{X}$. The design was a simple two-by-two factorial where $\mathrm{T}$ was conditioned either while $\mathrm{X}$ was undergoing extinction or not, and subsequently tested in the same context as where it was trained, or a different one. Participants received more extensive conditioning (12 trials) and Extinction (14 trials) to ensure that the learning in each phase was complete and that $\mathrm{X}$ was ambiguous in the sense of having acquired two associations.

The design also included three contexts. All training took place in Context A, and subjects received exposure to Contexts $\mathrm{B}$ and $\mathrm{C}$ during conditioning. Prior to test all participants received another brief exposure to Context $\mathrm{C}$. Thus, all participants received a context change prior to the test with T. Participants being tested in the same context as training, Context A, received a context-change procedure immediately before the test just as did the participants being tested in Context B, a

Table 2 Design of Experiment 2

\begin{tabular}{|c|c|c|c|}
\hline Group & Phase 1 & Phase 2 & \multicolumn{2}{|c|}{ Test } \\
\hline No Extinction-A & \multirow{4}{*}{ A: 12X+/B:--/C:-- } & \multirow{2}{*}{ A: -- / 4T+ } & C:-- I A: $4 \mathrm{~T}$ \\
\cline { 4 - 4 } & & C:-- I B: $4 \mathrm{~T}$ \\
\hline No Extinction-B & \multirow{2}{*}{ A: 14X- / 4T+ } & C:-- I A: $4 \mathrm{~T}$ \\
\hline With Extinction-A & & C:-- I B: 4T \\
\hline With Extinction-B & & &
\end{tabular}

Note A: and B: and C: are different galaxy contexts. $\mathrm{X}$ and $\mathrm{T}$ are red or yellow lights, counterbalanced. + and - refer to the presence and absence of an attack by an enemy spaceship, respectively. Numbers refer to the number of trials. - indicates exposure to the context. / indicates intermixed trials. | indicates a separation of trial types feature not present in the prior experiment (see Nelson et al. 2013, for discussion).

We used conditioning and extinction parameters similar to those found in Nelson et al. (2012), where asymptotic response levels were evident after 12 conditioning trials and 14 extinction trials. We also replaced the purple CS with red or yellow, as was used in Nelson et al. (2011b, 2013). Finally, we conducted only four conditioning trials with $\mathrm{T}$ as potentially weaker levels of conditioning have been associated with increased sensitivity to contextual changes (Hall and Honey 1990).

Method

\section{Subjects and apparatus}

Participants were volunteers solicited from a college campus. We sought to recruit 80 participants for this study, with minimum group sizes of 20 . Recruitment sheets allowed more participants than necessary to volunteer to compensate for "no shows." As no participant who volunteered and appeared for their appointment was turned away, 98 participants ultimately participated.

The same apparatus as was used in the previous experiment was used here. In addition to the Eagle 1 and Crab Nebula galaxy backgrounds (counterbalanced as Contexts A and B) an additional context ("Swirl" galaxy) was used as Context C, as described by Nelson et al. (2013). Red (RGB 255-0-0, appearing in third oval) and yellow sensors were counterbalanced as $\mathrm{X}$ and $\mathrm{T}$.

\section{Procedure}

Random assignment placed 23 participants in each of the No Extinction Groups and 24 and 28 participants in the With Extinction A and With Extinction B Groups, respectively.

Phase 1, $\mathrm{X}+$ During conditioning participants received trials in the three contexts in the order A,B,C,A,C,B, with the position of $\mathrm{A}$ and $\mathrm{B}$ in the sequence counterbalanced. With each exposure to A they received six trials where $\mathrm{X}$ was paired with the attack US. Participants simply played the game with no stimuli presented in contexts B and C for the same amount of time. The average ITI in this phase was $11 \mathrm{~s}$.

Phase 2, $X$-/T+ Extinction took place in Context A. All participants received a total of 18 trials in this phase, with an average ITI of $11 \mathrm{~s}$. Four of the trials (trials 5, 7, 12, and 18) consisted of pairing $\mathrm{T}$ with the attack US. The remaining 14 trials varied by Group. Groups Extinction A and Extinction B received presentations of $\mathrm{X}$ without the US on these trials. The 
other groups received presentations of the black sensor which produced no visual changes in the game.

\section{Testing with $T$}

Immediately following extinction, all participants received $60 \mathrm{~s}$ of exposure to Context $\mathrm{C}$ followed by a context change. Those in groups Extinction B and No Extinction B then received testing in Context B with the remainder of the groups tested in Context A. T was presented four times in extinction with an average ITI of $11 \mathrm{~s}$.

\section{Results}

Five participants were excluded for failing to condition to X. Data from training are shown in Fig. 3, which does not collapse across groups as there was one, seemingly spurious, difference among the groups in the first phase on trial four of conditioning. Suppression in the groups to be tested in Context A was slightly greater than that of those to be tested in Context B. Conditioning of T occurred uneventfully. On test, shown in Fig. 4, there was reliable extinction of responding, with some possibly weak evidence for less suppression in Context B than in Context A in the No Extinction Group. There was no evidence for an effect of Context in the With Extinction Group. There was no strong effect of the Extinction variable on test in either context, with the odds favoring the null hypotheses.

Phase 1: $X+$ trials Figure 3 shows the data from Conditioning and Extinction. Seven participants were excluded in Phase 1

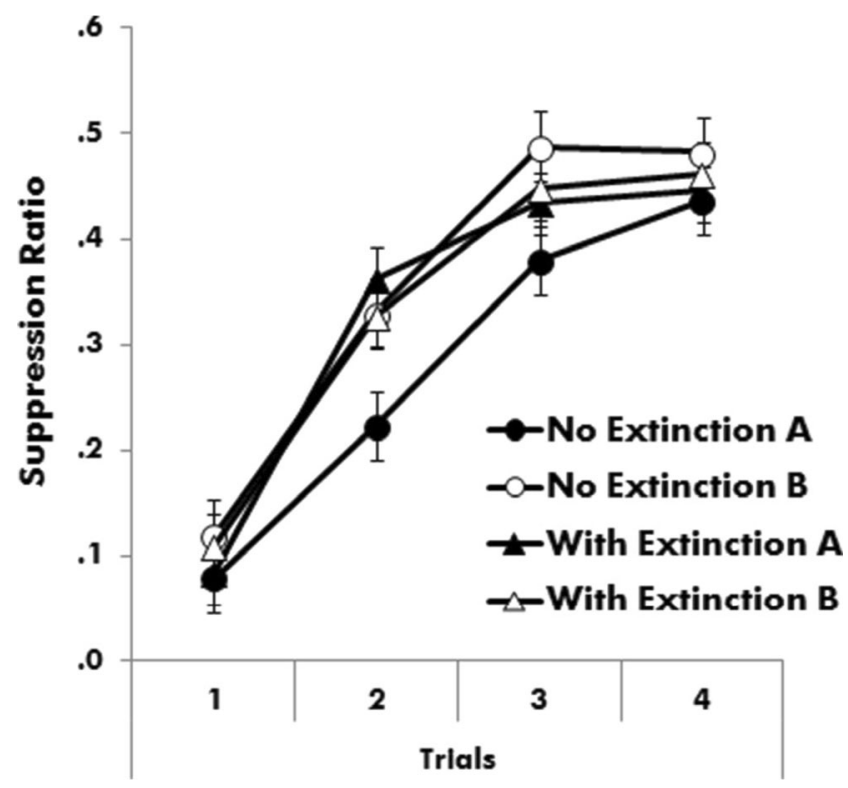

Fig. 4 Test data from Experiment 2. Points represent extinction trials with $\mathrm{T}$ on test in Context A where it was trained (solid symbols) or in Context B where it had never before been presented (open symbols). Participants had received conditioning with $\mathrm{T}$ either concurrently with extinction of X (triangles) or not (circles). Bars represent the standard error of the mean

due to occasional zero pre-CS responses. An Extinction (to be extinguished or not) by Context (to be tested in A or B) by Trials ANOVA of suppression to $\mathrm{X}$ showed an effect of trials, $F(11,902) 80.86,<.0001, \eta_{p}^{2}=.5$. Unexpectedly, there was a Context $\times$ Trials interaction that just passed the standard of reliability, $F(11,902)=1.83, p=.0453, \eta_{p}^{2}=.02$. There were no other effects, $F \mathrm{~s} \leq 1.02, p \mathrm{~s} \geq .31$. Simple effect tests of Context on each trial found a single difference on trial four,

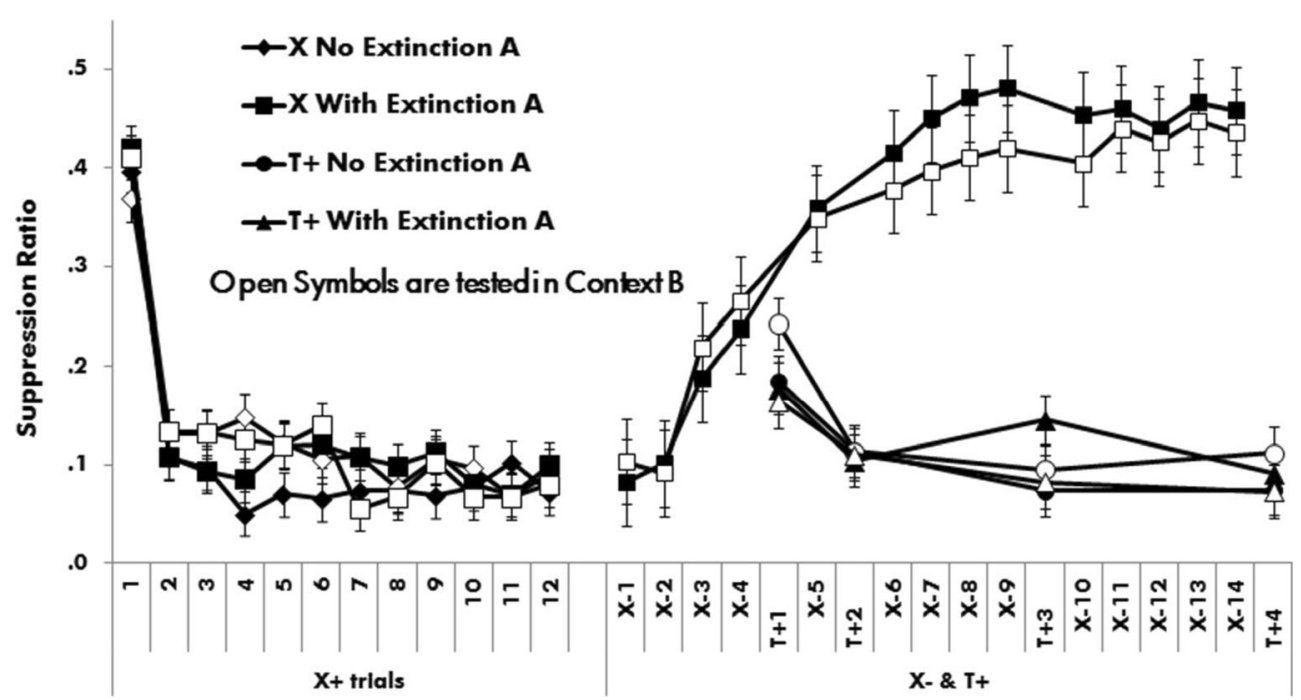

Fig. 3 Training data from Experiment 2. Acquisition of suppression to $X$ (squares and diamonds) during Phase 1 conditioning is shown at left. Extinction of X (squares) and conditioning of $\mathrm{T}$ (circles and triangles) during Phase 2 is shown at right. Axis labels (e.g., 1,2...) reflect trial numbers. Labels containing alphabetic text indicate both the type of trial (e.g., $\mathrm{T}+/ \mathrm{X}-$ ) and the trial number. + indicates trials where the $\mathrm{CS}$ was paired with an attack. - indicates trials without an attack. Bars represent the standard error of the mean 
$F(1,590)=9.32, p=.002, d=.66$. Suppression in the groups to be tested in Context A combined was slightly greater than that of those to be tested in Context B combined. There were no differences on any other trial, $F \mathrm{~s}(1,590) \leq 2.26, p \mathrm{~s} \geq .106$.

Phase 2: $X$-trials Data from Extinction are shown in Fig. 3, right-hand side. Eight subjects were removed due to zero preCS scores. The data were analyzed with a Context $x$ Trials ANOVA. There was a significant effect of Trials, $F(13,507)=51.06, p<.0001, \eta_{p}^{2}=.57$. There were no other effects, $F \mathrm{~s}<1$.

Phase 2: $T+$ trials Data from Conditioning of $\mathrm{T}$ are also shown in Fig. 3, right-hand side. Two subjects were removed due to zero pre-CS scores. The data were analyzed with an Extinction $\times$ Context $\times$ Trials ANOVA. There was an effect of trials, $F(3,261)=12.68, p<.0001, \eta_{p}^{2}=.13$. There were no other effects, Fs $<1.21, p s>.30$. The Extinction $\times$ Test Context interaction approached reliability, $F(1,87)=3.17, p=$ .079 . As a pre-existing difference here would confound our testing, we investigated these possible differences further. Among groups not receiving Extinction, responding in those to be tested in Context A averaged .11 and those tested in Context B averaged .14. Among those that did receive extinction, responding in those to be tested in Context $\mathrm{A}$ averaged .13 and those to be tested in Context B averaged .11. The largest difference between means was the difference between the No Extinction and With Extinction Groups in Context $\mathrm{B}$, which was not reliable, $F(1,346)=3.03, p=$ .08 . There were no reliable differences among the groups during conditioning of $\mathrm{T}$.

\section{Tests with $T$}

The data from this test are shown in Fig. 4. Five participants were eliminated for a zero pre-CS score on some trial. An Extinction $\times$ Context $\times$ Trials ANOVA showed an effect of Trials, $F(3,252)=166.63, p<.0001, \eta^{2} p=.66$. There were no other effects involving trials (i.e., Trials $\times$ Context, Trials $\times$ Extinction, and the three-way interaction), $F \mathrm{~s} \leq 1.84, p \mathrm{~s}>.14$, with the odds strongly favoring the null in every case (odds $\geq$ 327.18). The effect of Context approached significance, $F(1$, $84)=3.03, p=.085$, with no hint of an interaction with the Extinction variable, $F(1,84)=2.23, p=.14$. The odds slightly favored the null regarding the effect of Context (odds $=1.85$ ) and its interaction with Extinction (odds =2.84). The effects involving both Trials and Extinction were not significant, $F_{\mathbf{S}}<$ 1 , with the odds strongly favoring the null (odds $\geq 440$ ).

Despite the lack of an interaction, we investigated simple effects due to our clear a-priori predictions. Those analyses suggested an effect of Context in the No Extinction Group, $F(1,84)=5, p=.028, \eta_{p}^{2}=.06$, but none in the With Extinction Group, $F<1$, where the odds again provided positive evidence for the null (odds $=6.67$ ) regarding the Extinction variable. Averaged across trials there was no effect of the Extinction manipulation in either context, $F_{\mathrm{S}}<1$ with the odds weakly favoring the null in Context A (odds $=1.88$ ) and positively favoring it in Context $\mathrm{B}$ (odds $=5.61$ ).

Inspection of Fig. 4 suggests that any effect of Context that could be inferred, regardless of the strength of such an inference, was predominately due to greater suppression in Context A in the No Extinction Group. On Trial 2, that group differed from the other three groups, $F \mathrm{~s}(1,239)_{\text {min-max }}=5.24-9.49, p \mathrm{~s}$ ${ }_{\min -\max } .002-.023, d_{\min -\max }=.69-91$. We generally must interpret this result cautiously as it is not indicated by the overall analysis. To the extent that the effects suggested are due to greater suppression in this group, it indicates that extinction of $\mathrm{T}$ in the context of acquisition was simply somewhat less effective than in Context B or following Extinction of X. In any case, there is no support for the idea of enhanced attention to the context as evidenced by contextual control of $\mathrm{T}$ resulting from Extinction of $\mathrm{X}$.

\section{Discussion}

In the present experiment, asymptotic conditioning and extinction of X occurred. Moreover, in the group that received extinction of $\mathrm{X}$, conditioning trials with $\mathrm{T}$ took place both while extinction of $\mathrm{X}$ was occurring (i.e., $\mathrm{T}+$ trials 1 and 2 ) and after it had reached asymptote $(\mathrm{T}+$ trials 3 and 4$)$. The overall analysis of responding on test weakly $(p=.085)$ indicated that there was a context effect, and that it had the same effect on both groups. Further analysis based on a-priori expectations suggested an interaction. There was a relatively clearer suggestion of a context change effect in the groups that had not received extinction of X, and none could be observed in the group that did receive extinction of X. To the extent that this result is reliable, it would be exactly the opposite of what has previously been found in predictive learning methods.

It is possible that there was a "learning to learn" (LTL) effect with extinction. Extinction with one stimulus can enhance the rate at which extinction occurs with another in some situations (i.e., Vurbic and Bouton 2011). Such an effect would lead extinction of $\mathrm{T}$ to proceed more rapidly in the With Extinction Groups than in the No Extinction Groups. In Context A, this would result in the With Extinction Groups showing less suppression than the No Extinction Group, as the analyses suggest could have occurred. If the LTL effect was itself context specific in this method (though there is no evidence of such an effect where LTL of extinction has been observed, Vurbic and Bouton 2011), then no effect of context change would be observable. Both the With Extinction Groups would show less suppression than the No Extinction Groups tested in Context A, as was indicated by the simpleeffect tests. Whatever the merits of the overall reliability of the pattern of data observed and the explanation above, there was 
no evidence of enhanced contextual control of responding to $T$ as expected by ATCP.

Asymptotic extinction was reached in this experiment. We conducted asymptotic extinction to ensure that $\mathrm{X}$ was fully ambiguous in the sense of having two well-learned associations. Nevertheless, that manipulation problematically raises the possibility that participants were no longer attending to the context (see Leon et al. 2011) and the change in context had no effect. This possibility was ruled out in the first experiment by a renewal test, though the extinction in that experiment was pre-asymptotic. This possibility is also reduced by the results of Nelson et al. (2011a), who observed renewal with a stimulus receiving even more extinction. Nevertheless, the next experiment addressed the possibility that participants were no longer attending to the contexts.

\section{Experiment 3}

The design of Experiment 3 is shown in Table 3. In Experiment 3 participants received conditioning and extinction of $X$, with conditioning of T occurring during the extinction of $\mathrm{X}$ as in Experiment 2. All participants received testing of both $\mathrm{X}$ and $\mathrm{T}$, with the order of testing counterbalanced. Half the participants were tested in the same context as training and the other half were tested in a different context. The parameters were otherwise the same as in the previous experiment except that context $\mathrm{C}$ was not included in the design. On the first test, participants tested in Context A received the test uninterrupted, while those tested in Context $\mathrm{B}$ received the context switch. ATCP does not address what aspect of the contexts needs to be different, only that the contexts are different. Including the context change procedure itself as part of the context (as was done in Experiment 1) was intended to further enhance any contextual differences at test. The expectation was that there would be an increase in suppression observed to the extinguished $\mathrm{X}$ with a context change, and according to ACTP there should be a decrease in suppression to the simple excitor $\mathrm{T}$ with a context change.

Table 3 Design of Experiment 3

\begin{tabular}{|c|c|c|c|}
\hline Group & Phase 1 & Phase 2 & $\begin{array}{c}\text { Test } \\
\mathrm{T} \text { and } \mathrm{X} \text { test order } \\
\text { was counterbalanced } \\
\end{array}$ \\
\hline With Extinction-A & \multirow{2}{*}{ A: $12 \mathrm{X}+/ \mathrm{B}:--$} & \multirow{2}{*}{ A: $14 \mathrm{X}-/ 4 \mathrm{~T}+$} & A: 4T- A:4X- \\
\hline With Extinction-B & & & B: 4T- B:4X- \\
\hline
\end{tabular}

Method

\section{Subjects and apparatus}

Subjects were volunteers from a college campus. We planned on recruiting group sizes of 20 and, again, had good participant turnout resulting in a total of 105 volunteers. The same apparatus as was used in the previous experiments was used here. Context $\mathrm{C}$ was not used in the present experiment.

\section{Procedure}

The conditioning of $\mathrm{X}$, extinction of $\mathrm{X}$, and conditioning of $\mathrm{T}$ were conducted exactly as in the previous experiment except that there was no exposure to Context C. Half the participants in each group were tested in Context $\mathrm{A}$ and the other half were tested in Context B. There were two tests, one test with $\mathrm{X}$ and another test with T. Half of each of these groups first received four test trials with $\mathrm{X}$ followed by four test trials with $\mathrm{T}$, the other half had this arrangement reversed. Thus, there were four groups into which to assign participants (A-X-T, A-T$\mathrm{X}, \mathrm{B}-\mathrm{X}-\mathrm{T}, \mathrm{B}-\mathrm{T}-\mathrm{X})$ where the first letter refers to the context of testing and the next two letters designate the order in which $\mathrm{T}$ and $\mathrm{X}$ were tested. Random assignment placed 25, 27, 27, and 26 participants into groups A-X-T, B-X-T, A-X-T, and A$\mathrm{T}-\mathrm{X}$, respectively. The ITIs on each test were the same as used on test in the previous experiment.

Results

Suppression to $\mathrm{X}$ was acquired and extinguished equivalently between the different groups. Suppression to T was likewise acquired equivalently. The data are shown in Fig. 5, which collapses across the insignificant grouping variables. On test, shown in Fig. 6, there was renewal to $\mathrm{X}$ indicating that the contexts were not being ignored. There was weak evidence of a context-switch effect on $\mathrm{T}$.

\section{Phases 1 and 2}

Data in both phases were analyzed with Context (to be tested in A or B) by Trials ANOVAs. In analyzing suppression to X screening for zero pre-CS scores eliminated three participants from the Phase 1 analyses and 14 from Phase 2. In Phase 1 there was an effect of Trials, $F(11,1023)=79.64, p<.0001$, $\eta_{p}^{2}=.46$, and no other effects, $\mathrm{Fs}<1$. In Phase 2, there was an effect of Trials as $\mathrm{X}$ underwent extinction, $F(13,1066)=$ $59.67, p<.0001, \eta_{p}^{2}=.42$, and no other effects, Fs $<1$. When analyzing suppression to T, screening of the pre-CS data eliminated one participant. There was an effect of Trials as suppression was acquired, $F(3,285)=16.16, p<.0001, \eta^{2}{ }_{p}=.15$, and no other effects, $F \mathrm{~s}<1$. 


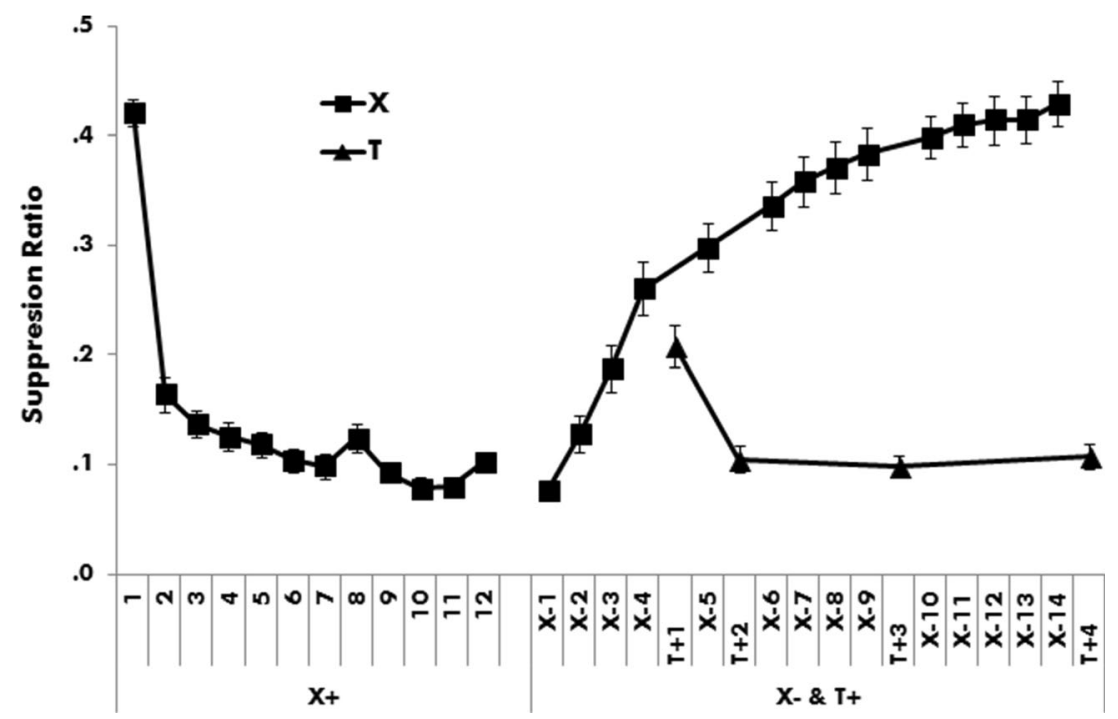

Fig. 5 Training data from Experiment 3. Suppression to $X$ during Phase 1 conditioning, Phase 2 extinction, and suppression to T during Phase 3. Extinction of X (squares) and conditioning of T (triangles) during Phase 2 is shown at right. Axis labels (e.g., 1,2 ...) reflect trial numbers. Labels

\section{Testing}

Screening for zero pre-CS rates eliminated seven participants. Data from testing are shown in Fig. 6, which collapses across the Test Time variable as it had no interactions with the Context effect.

Renewal test with $X$ The four test trials with $\mathrm{X}$ were analyzed with a Context $\times$ Test Time $\times$ Trials ANOVA. There was an effect of Trials, $F(3,261)=43.39, p<.0001, \eta_{p}^{2}=.33$, and a Trials $\times$ Test Time interaction, $F(3,261)=13.72, p<.0001$,

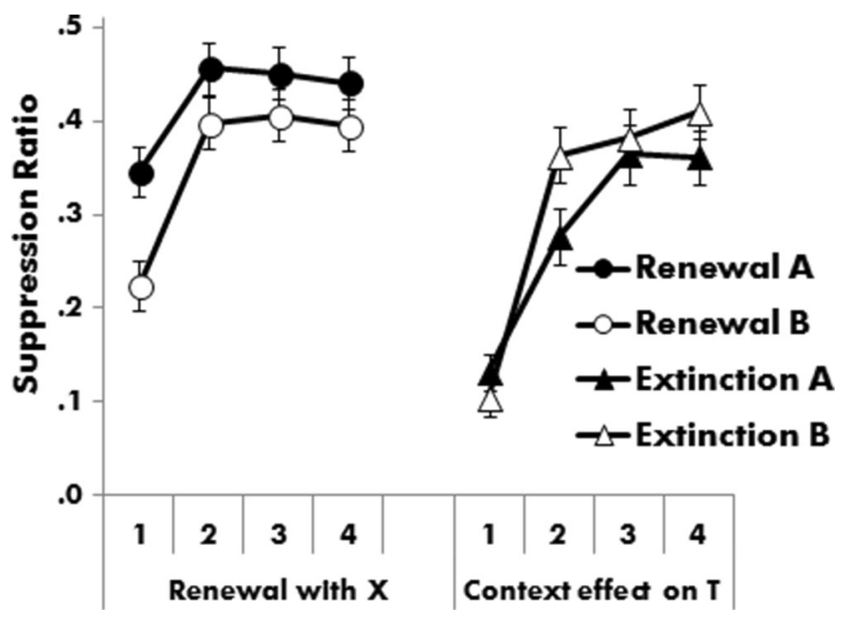

Fig. 6 Test data from Experiment 3. Left panel shows suppression to X test in Context A (solid circles) where extinction of X had occurred or Context B (open circles) where X had never before been presented. Right panel shows responding to $\mathrm{T}$ in Context A where it had been trained (solid triangles) or Context B where it had never before been presented (open triangles). Bars represent the standard error of the mean. See text for details containing alphabetic text indicate both the type of trial (e.g., T+/X-) and the trial number. + indicates trials where the conditioned stimulus (CS) was paired with an attack. - indicates trials without an attack. Bars represent the standard error of the mean

$\eta_{p}^{2}=.13$. Testing $\mathrm{X}$ after $\mathrm{T}$ led to somewhat more suppression overall to $\mathrm{X}$ on the early trials, which thus underwent greater extinction over the test trials.

Of most importance, there was a Context $\times$ Trials interaction, $F(3,261)=3.01, p=.03, \eta_{p}^{2}=.03$, that did not interact with Test Time $F=.12$. The main effect of Context approached significance, $F(1,87)=3.92, p=.0508$, and there was no interaction with Test Time, $F=.14$. Simple effects of Context at each trial showed reliably more suppression in Context $\mathrm{B}$ than in Context $\mathrm{A}$ on Trial $1, F(1,141)=$ 9.94, $p=.001, d=.66$, and none on other trials, $F \mathrm{~s}(1$, $141) \leq 2.3, p \mathrm{~s} \geq .13$.

Context effect with $T$ The four test trials with $T$ were analyzed with a Context $\times$ Test Time $\times$ Trials ANOVA. There was a reliable effect of Trials, $F(3,261)=65.09, p<.0001, \eta^{2}{ }_{p}=.43$. The Trials $\times$ Context interaction only approached significance, $F(3,261)=2.49, p=.061, \eta_{p}^{2}=.028$. There were no other effects, $F \mathrm{~s} \leq 2.35, p \mathrm{~s} \geq .12$. The odds were in favor of the null in all effects involving Context (odds $\min _{\text {max }}=2.82$ 240.9). Given our a-priori interest in a context effect here, we nevertheless conducted simple effect tests on each trial. These tests potentially showed less suppression in Context B than in Context A on trial 2, $F(1,219)=7.81, p=.0057, d=$ .59 , and no other trial, $F_{\mathrm{S}}(1,219) \leq 2.35, p \mathrm{~s} \geq .127$.

\section{Discussion}

The present experiment produced evidence that even after asymptotic extinction, participants were still sensitive to context changes. Renewal with X was evident. In ATCP terms, participants were still attending to the context. Nevertheless, 
the effect of a context change on responding to the excitor $\mathrm{T}$ was negligible, if present at all. Unfortunately, we did not have a control condition where the context switch effect on $T$ was assessed in a group that had not received extinction of $\mathrm{X}$ as in Experiment 2, and the ambiguity in interpretation produced by these results highlights the need for such a control. The possible effect observed with $\mathrm{T}$ might simply represent an ordinary small effect of a context change with this method (see Nelson et al. 2011b) rather than the specific effect of prior extinction. Experiment 4 was conducted to provide the appropriate control.

\section{Experiment 4}

Experiment 4 used the same $2 \times 2$ factorial design of Experiment 2 (see Table 4) but Context $\mathrm{C}$ was omitted as in Experiment 3 where evidence of attention to contexts was obtained with the renewal test. All participants received conditioning of $\mathrm{X}$. T was then conditioned while $\mathrm{X}$ was undergoing extinction or not, and subsequently tested in Context A or B. The expectation at test, based on ACTP, is that any effect of Extinction observed in Context $\mathrm{B}$, where $\mathrm{T}$ has never before been presented, should be greater than in Context A.

\section{Method}

\section{Subjects and apparatus}

Subjects were volunteers from a college campus. The participant pool for this experiment was expected to be smaller than that of the others due to the time frame being near the end of an academic year, thus we planned to collect data from 48 participants as reliable effects of renewal occur with this method using similar N (Nelson et al. 2011a). Attendance was surprisingly good and 51 volunteers ultimately participated. Random assignment placed 12 in Groups No Extinction A and Extinction B, 14 in No Extinction B, and 13 in Extinction A.

Table 4 Design of Experiment 4

\begin{tabular}{|c|c|c|c|}
\hline Group & Phase 1 & Phase 2 & Test \\
\hline No Extinction-A & \multirow{4}{*}{ A: $12 \mathrm{X}+/ \mathrm{B}:--$} & \multirow{2}{*}{ A: $--/ 4 \mathrm{~T}+$} & A: $4 \mathrm{~T}$ \\
\hline No Extinction-B & & & B: 4T \\
\hline & & \multirow{2}{*}{ A: $14 \mathrm{X}-/ 4 \mathrm{~T}+$} & \\
\hline With Extinction-B & & & B: 4T \\
\hline \multicolumn{4}{|c|}{$\begin{array}{l}\text { Note A: and B: are different galaxy contexts. } \mathrm{X} \text { and } \mathrm{T} \text { are red or yellow } \\
\text { lights, counterbalanced. + and - refer to the presence and absence of an } \\
\text { attack by an enemy spaceship, respectively. Numbers refer to the number } \\
\text { of trials. - indicates exposure to the context. / indicates intermixed trials. } \\
\text { indicates a separation of trial types. * A programming error altered these } \\
\text { events such that there were } 13 \mathrm{X} \text { - trials, } 1 \mathrm{X}+\text { trial, and } 3 \mathrm{~T}-\text { trials. See text } \\
\text { for details }\end{array}$} \\
\hline
\end{tabular}

The same video game as was used in the previous experiment was used here.

\section{Procedure}

The procedure and parameters were the same as in Experiment 2 with two exceptions. First, Context $C$ was not used. Second, there was a programming error in the With Extinction groups. In these groups the second scheduled $\mathrm{T}+$ trial was inadvertently programmed as an $\mathrm{X}+$ trial. Thus, these groups received one fewer $\mathrm{T}+$ trial than did the No Extinction conditions, and two phases of Extinction (before and after the inadvertent $\mathrm{X}+$ trial). Despite that confound, the error has interesting implications for the interpretation of the results.

Results

The data from the first two phases of training are shown in Fig. 7. No participant was eliminated for failing to condition to $\mathrm{X}$. Conditioning of $\mathrm{X}$ occurred equally in all groups. Extinction in the With Extinction Groups was interrupted after the fifth extinction trial. Nevertheless, extinction occurred both before and after that error. Despite the fact that the With Extinction Groups received one less $\mathrm{T}+$ trial, suppression to $\mathrm{T}$ was acquired equally in all groups. On test, shown in Fig. 8, there was a main effect of Context. There was less suppression to $\mathrm{T}$ in Context $\mathrm{B}$ than in Context $\mathrm{A}$ in both groups. There was no measurable effect of the Extinction manipulation, with the odds providing positive to very strong support for the null in all tests involving the Extinction manipulation. The following analyses support these descriptions.

Phase 1: $X+$ No participant was eliminated for having a zero pre-CS in Phase 1. Conditioning with $X$ was analyzed with an Extinction (to be extinguished or not) $\times$ Test Context (to be tested in context A or B) ANOVA. There was a significant effect of Trials, $F(13,260)=47.03, p<.0001, \eta_{p}^{2}=.5$, and no effects involving Test Context, $F_{\mathrm{s}} \leq 1.24, p \mathrm{~s} \geq .24$. The data are shown in Fig. 7, which collapses across the insignificant Test Context variable Fig. 8.

Phase 2: $X$ - As reported in the opening summary, the groups receiving extinction of $\mathrm{X}$ inadvertently received a reinforced $\mathrm{X}$ trial on what should have been the second $\mathrm{T}+$ trial (above $\mathrm{T}$ $+2 / \mathrm{X}+$ in Fig. 7). There were three subjects excluded for at least one trial with a zero pre-CS in this phase, and all three were in the group to subsequently be tested in Context $\mathrm{B}$. The exclusion was not necessarily independent of the four groups in the experiment in the analysis of this phase, $X^{2}(3)=10.36$, $p=.02$. Analyses excluding those subjects, or including those subjects but excluding trials 2 and 4 where the zero pre-CS scores occurred, reached exactly the same conclusions. For brevity, we will report the only the former. 


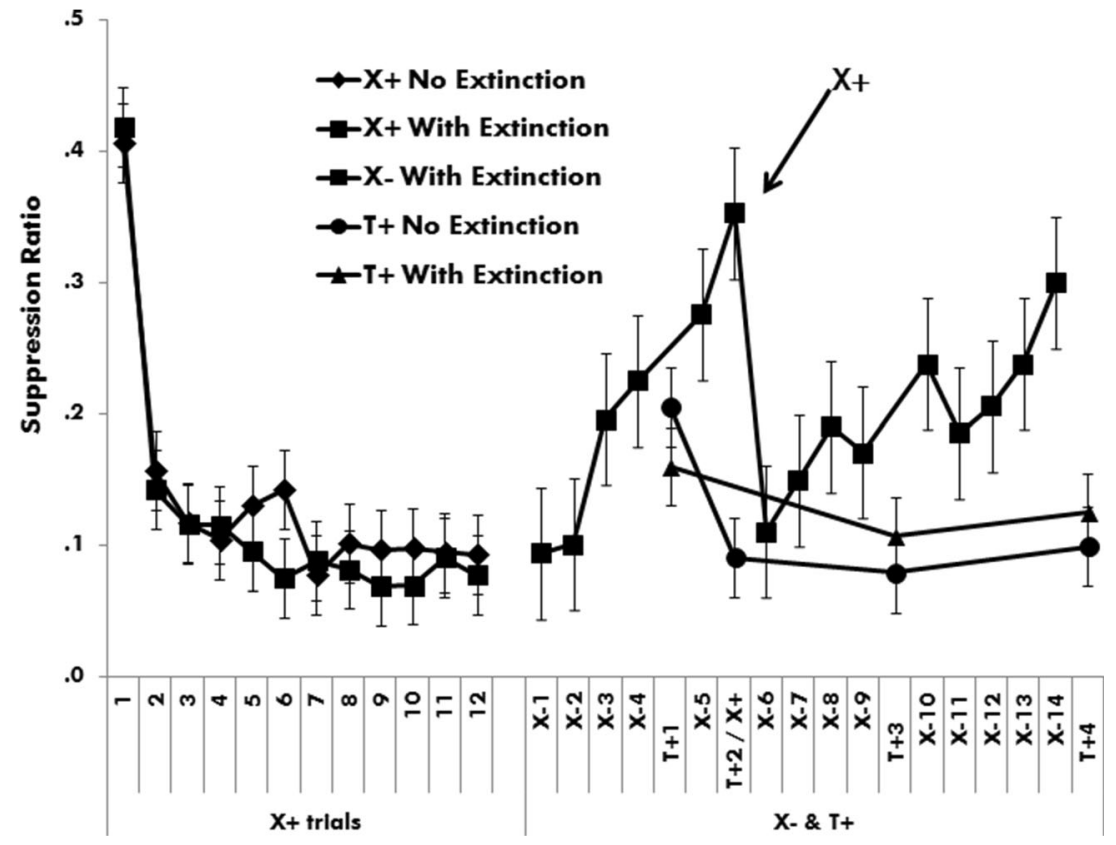

Fig. 7 Training data from Experiment 4. Acquisition of suppression to $X$ (squares $=$ With Extinction Groups and diamonds $=$ No Extinction Groups) during Phase 1 conditioning is shown at left. Extinction of $\mathrm{X}$ (squares) and conditioning of T (triangles and circles) during Phase 2 is shown at right. Axis labels (e.g., 1,2...) reflect trial numbers. Labels

A Test Context $\times$ Trials ANOVA over the $14 \mathrm{X}$ - trials revealed an effect of Trials, $F(13,260)=3.02, p<.0001, \eta_{p}^{2}=$ .13 , and no effects of Test Context, $F \mathrm{~s} \leq 2.31, p \mathrm{~s} \geq .14$. The inadvertent $\mathrm{X}+$ trial after trial 5 led to two apparent extinction

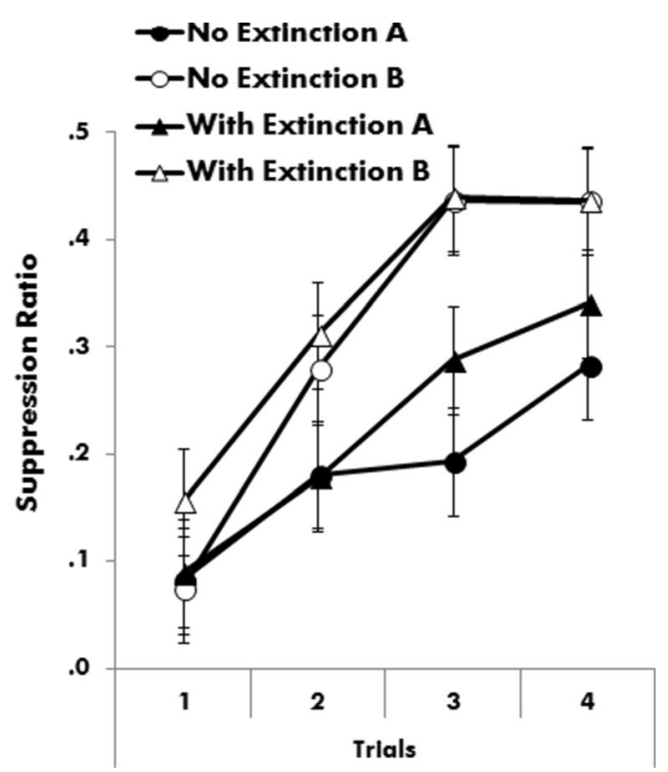

Fig. 8 Test data from Experiment 4. Points represent extinction trials with $\mathrm{T}$ on test in Context A where it was trained (solid symbols) or in Context B where it had never before been presented (open symbols). Participants had received conditioning with $\mathrm{T}$ either concurrently with extinction of X (triangles) or not (circles). Bars represent the standard error of the mean

containing alphabetic text indicate both the type of trial (e.g., $\mathrm{T}+\mathrm{X}-$-) and the trial number. + indicates trials where the conditioned stimulus (CS) was paired with an attack. - indicates trials without an attack. Bars represent the standard error of the mean

curves. Suppression decreased from trials $1-5, F(4,84)=4.92$, $p=.001, \eta_{p}^{2}=.19$, and again from trials $6-14, F(8,168)=$ $3.85, p=.0003, \eta_{p}^{2}=.14$.

Phase 2: T+ One subject was eliminated for a zero pre-CS score. Because the With Extinction group was missing the second $\mathrm{T}+$ trial, only the first, third, and fourth $\mathrm{T}+$ trials were analyzed with an Extinction $\times$ Test Context $\times$ Trials ANOVA. The analysis showed an effect of Trials, $F(2,94)=7.78, \mathrm{p}<$ $.0001, \eta_{p}^{2}=.14$, and no other effects, $F_{\mathrm{s}}<1$.

Test with $T$ in $A$ or $B$

The data from these tests are shown in Fig. 6. Two subjects were removed due to zero-pre CS scores. The four test trials were analyzed with an Extinction $\times$ Context $\times$ Trials ANOVA. The analysis revealed an effect of trials as responding extinguished over the test, $F(3,135)=34.71, p<.0001, \eta^{2} p$ $=.44$. There was a main effect of Context, with less responding in Context $\mathrm{B}$ than in Context $\mathrm{A}, F(1,45)=$ $10.01, p=.003, d=.84$. There were no other effects, $F \mathrm{~s}<1$. The odds favored the null regarding the effect of Extinction (odds $=4.02)$, the Extinction $\times$ Context interaction (odds $=$ 9.46), and very strongly favored the absence of a three-way interaction of Extinction $\times$ Context $\times$ Trials (odds $=374.76$ ) .

Simple-effect tests confirmed that there was clearly no interaction of prior Extinction with the contextual control. The effect of Context was present in both the No Extinction Group, $F(1,132)=5.32, p=.03, d=.94$, and the With Extinction 
Group, $F(1,132)=4.69, p=.036, d=.87$. There was no measurable effect of the Extinction manipulation in either Context, $F \mathrm{~S}<1$, with the odds positively supporting the null both in Context A (odds 3.82) and in Context B (odds = 5) where an effect was expected.

There was room to see an effect of Extinction in Context B as responding on the first two trials was clearly lower than on the last two, showing apparent room to observe differences. The first trial, which is empirically the most sensitive to context effects after extinction, had the greatest range in which to observe a decrease in suppression. The first trial in the With Extinction Group tested in B averaged .155 with a standard error of .052. Thus, a mean of zero (complete suppression) is clearly not within a $95 \%$ confidence interval. That observation supports that suppression was not embedded so deeply into a floor that differences could not be seen.

The lack of effect of the Extinction manipulation here is not due to a power issue, even given that the sample sizes were smaller than in the previous experiments. The preset pattern of means would require an approximate $450 \%$ increase in $\mathrm{N}$ for effects of, or involving, Extinction $\times$ Context to be significant. In such a case, the effect would be in the opposite direction as predicted by ATCP. The effect size for Context in the No Extinction Group is numerically larger than in the Extinction Group, which would be consistent with the pattern observed in Experiment 2.

\section{Discussion}

In the present experiment participants underwent conditioning with $\mathrm{T}$ either while $\mathrm{X}$ was undergoing extinction or not. A programming error introduced a confound between the Extinction conditions where the group receiving conditioning with Extinction received 1 less $\mathrm{T}+$ trial. If anything, that one less trial would be expected to enhance the stimulus' sensitivity to contextual changes (e.g., Hall and Honey 1990). Also, the error introduced a conditioning trial with $\mathrm{X}$ so that $\mathrm{X}$ essentially underwent two phases of extinction. There was extinction of $\mathrm{X}$ prior to the reinforced trial that reinstated high suppression, and extinction of $\mathrm{X}$ after that trial. According to ATCP these conditions should make the meaning of $X$ especially uncertain, ensuring especially high levels of attention to the context. Nevertheless, on test the context-switch effect on $\mathrm{T}$ in the group that received extinction of $\mathrm{X}$ was no different from that in the group that had not received extinction of X.

We could argue that because a context effect was observed in the No Extinction Groups, attention was being devoted to the contexts throughout the experiment regardless of the Extinction manipulation and extended training. The exact nature of the relationship between levels of attention and levels of contextual control is not explicitly specified in ACTP. That is, there is no direct statement as to whether contextual control is achieved to its maximum degree given any level of attention in an all-or-none fashion, or if contextual control is a graded function of levels of attention. If we assume either that attention was maintained as its highest level or that contextual control is an all-or-none function of any level of attention, then there would be no room for increases and no effect of Extinction would be observed in the With Extinction Groups. We cannot rule out such a possibility. Nevertheless, a null effect of the Extinction manipulation was observed both in situations where a context switch affected (Experiment 4) and did not affect (Experiment 2) responding, suggesting relative independence of the two functions with the present procedures. Assuming asymptotic attention to the context also prompts the question as to why renewal was observed in Experiments 1 and 3, the effect for which ACTP was designed to explain. If asymptotic attention or contextual control was maintained throughout the experiment resulting in conditioning being context specific, and extinction being context specific, little effect of a context change should be expected (i.e., the opposing tendencies would cancel) unless second learning is especially sensitive to contextual control as suggested by Bouton (1993) and Nelson (2002).

\section{General discussion}

ATCP (e.g., Rosas and Callejas-Aguilera 2006) predicts that extinction of one stimulus will lead to contextual control of learning about other stimuli by way of extinction producing ambiguity and enhancing attention to the contexts. In humans, the result has been demonstrated only in predictive learning tasks. The present research investigated whether or not such an effect would occur in a behavioral task. Using a task that produces evidence of attention to contexts, as well as a variety of context-switch effects, no evidence for enhanced contextual control of learning by extinction of another stimulus was found.

Experiment 1 used conditioning and extinction parameters with stimulus $\mathrm{X}$ that are known to produce evidence consistent with enhanced attention to the context (Nelson et al. 2013). Nevertheless, subsequent conditioning to stimulus $T$ was no more affected by a context change when preceded by extinction of $\mathrm{X}$ than when not. Though a null result, the result carries weight because renewal with $\mathrm{X}$ was demonstrated at the same time as when $\mathrm{T}$ was undergoing conditioning. Thus, at the time of encoding $\mathrm{T}$ there was evidence that attention was maintained to the contexts according to ATCP. Also, renewal with $\mathrm{X}$ was demonstrated at the time of testing with $\mathrm{T}$, thus at the time $\mathrm{T}$ was tested there was evidence of attention to the contexts.

Experiments 2-4 used parameters that produced strong and asymptotic conditioning and extinction with $\mathrm{X}$. Conditioning of $\mathrm{T}$ was conducted while $\mathrm{X}$ was undergoing extinction in a manner analogous to other demonstrations with predictive- 
learning methods (e.g., Rosas and Callejas-Aguilera 2006). In no case was there convincing evidence of extinction of $\mathrm{X}$ enhancing contextual control of T, even when there was evidence of attention to the contexts at the time $\mathrm{T}$ was tested.

There are many differences between the task used here and predictive learning tasks that could relate to the divergent results. Predictive learning tasks involve more cognitive effort, participants experience the events in third person without personal consequence, and the dependent variables are more related to belief than action. In the suppression task participants read instructions at the beginning of the task which undoubtedly can affect subsequent performance. However, from that point on all stimuli are presented literally. Pairings of stimuli are visually experienced and have behavioral consequences to the participant. In predictive-learning tasks images of the stimuli (e.g., food, restaurants) that another individual has experienced might be presented. However, they are accompanied by labeling text that describes the pairing and personal consequences of those pairings, to the participant, as absent. Predictive ratings reflect a participant's beliefs, which may or may not change depending upon whether belief-based action is required. The results of predictive learning tasks are known to be sensitive to the way in which the questions used to gather the ratings are phrased (e.g., Matute et al. 1996). Questions regarding a participant's likelihood to act on their knowledge may be differentially sensitive to the prior-extinction manipulation than questions about belief in predictive relationships. Whether or not any of these factors relate to the discrepancy between our results and those obtained with predictivelearning techniques is unknown, but they are likely candidates.

Research with animals has demonstrated the effect of extinction contributing to the contextual control of simple conditioning. Such an effect in animals argues to its generality, making the null results presented here appear all the more unique. The effect with animals, however, may not be as assured as it appears. In a taste-aversion paradigm, Rosas and Callejas-Aguilera (2007) gave rats conditioning and extinction with flavor $\mathrm{X}$, or unpaired presentations of $\mathrm{X}$ and the lithium chloride injection. Then, $\mathrm{X}$ was presented alone in both groups where it underwent extinction in the former group, and was simply exposed in the latter as no conditioning had previously occurred. Following that phase, flavor Y was conditioned and then tested in the same or a different context as it was trained. The paper reports a context effect where there was less of an aversion to $\mathrm{Y}$ when tested in a different context than in the same context, and no such effect was present in the group for which $\mathrm{X}$ was never conditioned, and thus never extinguished. The effect of prior extinction, however, was not directly assessed. The paper does not report a difference between the extinction conditions when tested in Context $\mathrm{B}$ that is absent in Context A. Inspection of their figures shows the contrary. In the training context, A, there was what appeared to be a substantially stronger aversion in the group that received extinction than in the group that had received the unpaired X-US presentations for which extinction had not taken place prior to conditioning of $\mathrm{Y}$ (see Nelson et al. 2011b for further discussion).

Reports showing a response decrement with a context change with animals have all, at one point or other, involved thirsty rats with the use of liquids as either a CSs or reinforcers. Work with conditioning methods based on hunger and food consumption has shown no such effect (Nelson et al. 2011a). If any strong conclusion can be reached with Nelson et al.'s data, it would be that extinction of one CS enhanced transfer of responding with another CS to a different context. Such a finding could imply contextual control, but not of the nature being suggested by the work of Rosas and Callejas-Aguilera (2007) and the implications are further discussed in Nelson et al. (2011b). The work with animals where effects have been reported either lack comparisons where the effect of extinction is assessed at each level of the context manipulation (Bernal-Gamboa et al. 2013; Rosas and Callejas-Aguilera 2007), which would provide the most direct evidence for the effect, or are absent a No-Extinction control against which to ensure that the observed effects of context would not simply happen anyway (Bernal et al., Experiment 2; Bernal-Gamboa et al. 2014). Like the results of Nelson et al. (2011a) do with animals, the present results appear to place constraints on the generality of ACTP as an account of contextual control in humans.

Acknowledgments The methods and research presented here were made possible by Grant No. PSI2011-24231 from the Spanish Ministry of Science and Innovation and Grant No. IT-694-13 from the Basque government. Participation of Jeffrey A. Lamoureux in the project was supported by the Psychology Department of Boston College.

\section{References}

Bernal-Gamboa, R., Callejas-Aguilera, J. E., Nieto, J., \& Rosas, J. M. (2013). Extinction makes conditioning time-dependent. Journal of Experimental Psychology: Animal Behavior Processes, 39, 221232.

Bernal-Gamboa, R., Rosas, J. M., \& Callejas-Aguilera, J. E. (2014). Experiencing extinction within a task makes nonextinguished information learned within a different task context-dependent. Psychonomic Bulletin and Review, 3, 803-808.

Bouton, M. E. (1993). Context, time, and memory retrieval in the interference paradigms of Pavlovian learning. Psychological Bulletin, $114,80-99$.

Bouton, M. E. (1997). Signals for whether versus when an event will occur. In M. S. Fanselow \& M. E. Bouton (Eds.), Learning, motivation, and cognition: The functional behaviorism of Robert $C$. Bolles (pp. 385-409). Washington, DC: American Psychological Association.

Bouton, M. E. (2004). Context and behavioral processes in extinction. Learning \& Memory, 11, 485-494. 
Bouton, M. E., \& Bolles, R. C. (1979). Contextual control of the extinction of conditioned fear. Learning and Motivation, 10, 445-466.

Gawronski, B., \& Cesario, J. (2013). Of mice and men: What animal research can tell us about context effects on automatic responses in humans. Personality and Social Psychology Review, 17, 187-215.

Gawronski, B., Rydell, R. J., Vervliet, B., \& De Houwer, J. (2010). Generalization versus contextualization in automatic evaluation. Journal of Experimental Psychology: General, 139, 683-701.

Glover, S., \& Dixon, P. (2004). Likelihood ratios: A simple and flexible statistic for empirical psychologists. Psychonomic Bulletin \& Review, 11, 791-806.

Hall, G., \& Honey, R. C. (1990). Context-specific conditioning in the conditioned-emotional-response procedure. Journal of Experimental Psychology: Animal Behavior Processes, 16, 271-278.

Howell, D. C. (1987). Statistical methods for psychology. Boston, Duxbury.

Leon, S. P., Abad, M. J. F., \& Rosas, J. M. (2011). Context-outcome associations mediate context-switch effects in a human predictive learning task. Learning and Motivation, 42, 84-98.

Lindley, D. V. (1957). A statistical paradox. Biometrika, 44, 187-192.

Masson, M. E. J. (2011). A tutorial on a practical Bayesian alternative to null-hypothesis significance testing. Behavior Research, 43, 679-690.

Matute, H., Arcediano, F., \& Miller, R. R. (1996). Test questions modulate cue competition between causes and between effects. Journal of Experimental Psychology: Learning, Memory, and Cognition, 22, $182-196$.

Nelson, J. B. (2002). Context specificity of excitation and inhibition in ambiguous stimuli. Learning and Motivation, 33, 284-310.

Nelson, J. B. (2009). Contextual control of first- and second-learned excitation and inhibition in equally ambiguous stimuli. Learning \& Behavior, 37, 95-106.

Nelson, J. B. (2013) Mechanisms of extinction in emotional regulation. In. Rime, B., Mesquitea, B., \& Hermans, D (Eds.) Changing Emotions (pp. 60-66) Sussex, United Kingdom: Psychology Press, Taylor Francis Group.

Nelson, J. B., \& Bouton, M. E. (2002). Extinction, inhibition, and emotional intelligence. In L. Feldman-Barrett \& P. Salovey (Eds.), The wisdom in feeling: psychological processes in emotional intelligence (pp. 60-85). New York: Guilford Press.

Nelson, J. B., \& Callejas-Aguillera, J. E. (2007). The role of interference produced by conflicting associations in contextual control. Journal of Experimental Psychology: Animal Behavior Processes, 33, 314-326.

Nelson, J. B., \& Sanjuan, M. C. (2006). A context-specific latent-inhibition effect in a human conditioned-suppression task. Quarterly Journal of Experimental Psychology, 59, 1003-1020.
Nelson, J. B., \& Sanjuan, M. C. (2008). Flattening generalization gradients, context, and perceptual Learning. Learning \& Behavior, 36, 279-289.

Nelson, J. B., \& Sanjuan, M. C. (2009). Perceptual learning in a human conditioned suppression task. International Journal of Comparative Psychology, 22, 206-220.

Nelson, J. B., Lombas, S., \& Leon, S. P. (2011a). Concurrent extinction does not render appetitive conditioning context specific. Learning \& Behavior, 39, 87-94.

Nelson, J. B., Sanjuan, M. C., Vadillo-Ruiz, S., Perez, J., \& Leon, S. P. (2011b). Experimental renewal in humans. Journal of Experimental Psychology: Animal Behavior Processes, 37(1), 58-70.

Nelson, J. B., Gregory, P., \& Sanjuan, M. C. (2012). The extinction context enables extinction performance after a change in context. Behavioural Processes, 90, 372-377.

Nelson, J. B., Lamoureux, J. A., \& Leon, S. (2013). Extinction arouses attention to the context in a behavioral suppression task with humans. Journal of Experimental Psychology: Animal Behavior Processes, 39(1), 99-105.

Raftery, A. E. (1995). Bayesian model selection in social research. In P. V. Marsden (Ed.), Sociological methodology (pp. 111-196). Cambridge, MA: Blackwell.

Rosas, J. M., \& Callejas-Aguilera, J. E. (2006). Context switch effects on acquisition and extinction in human predictive learning. Journal of Experimental Psychology: Learning, Memory, and Cognition, 32, 461-474.

Rosas, J. M., \& Callejas-Aguilera, J. E. (2007). Acquisition of a conditioned taste aversion becomes context dependent when it is learned alter extinction. Quarterly Journal of Experimental Psychology, 60, 9-15.

Rosas, J. M., Callejas-Aguilera, J. E., Ramos-Álvarez, M. M., \& Abad, M. J. F. (2006a). Revision of retrieval theory of forgetting: what does make information context-specific? International Journal of Psychology and Psychological Therapy, 6, 147-166.

Rosas, J. M., García-Gutiérrez, A., \& Callejas-Aguilera, J. E. (2006b). Effects of context change upon retrieval of first and second learned information in human predictive learning. Psicológica, 27, 35-56.

Vurbic, D., \& Bouton, M. E. (2011). Secondary extinction in Pavlovian fear conditioning. Learning \& Behavior, 39, 202-211.

Wagenmakers, E. (2007). A practical solution to the pervasive problems of $p$ values. Psychonomic Bulletin and Review, 14, 779-804.

Ye, Y., \& Gawronski, B. (2014). Contextualization of mental representations and evaluative responses: A theory-based analysis of cultural differences. In J. Spencer-Rodgers \& K. Peng (Eds.), The psychological and cultural foundations of east Asian Cognition: Contradiction, change, and holism (pp. 1-11). New York: Oxford University Press. 